\title{
Reducing research waste in benign gynaecology and fertility research
}

DOI:

10.1111/1471-0528.14438

\section{Document Version}

Accepted author manuscript

Link to publication record in Manchester Research Explorer

\section{Citation for published version (APA):}

Duffy, J. M. N., Bhattacharya, S., Herman, M., Mol, B., Vail, A., Wilkinson, J., Farquhar, C., \& Cochrane

Gynaecology and Fertility Group (2017). Reducing research waste in benign gynaecology and fertility research.

BJOG: An International Journal of Obstetrics and Gynaecology, 124(3), 366-369. https://doi.org/10.1111/1471-

0528.14438

\section{Published in:}

BJOG: An International Journal of Obstetrics and Gynaecology

\section{Citing this paper}

Please note that where the full-text provided on Manchester Research Explorer is the Author Accepted Manuscript or Proof version this may differ from the final Published version. If citing, it is advised that you check and use the publisher's definitive version.

\section{General rights}

Copyright and moral rights for the publications made accessible in the Research Explorer are retained by the authors and/or other copyright owners and it is a condition of accessing publications that users recognise and abide by the legal requirements associated with these rights.

\section{Takedown policy}

If you believe that this document breaches copyright please refer to the University of Manchester's Takedown Procedures [http://man.ac.uk/04Y6Bo] or contact uml.scholarlycommunications@manchester.ac.uk providing relevant details, so we can investigate your claim.

\section{OPEN ACCESS}




\section{Reducing research waste in benign gynaecology and fertility}

\section{research}

JMN Duffy ${ }^{1,2}$ MBChB MRes BSc (Hons) PG HCL, S Bhattacharya ${ }^{3}$ FRCOG MD, M Herman ${ }^{4}$ MD PhD, B

Mol $^{5}$ MD PhD, A Vail ${ }^{6}$ BSC MSc, J Wilkinson ${ }^{6}$ BSC MSC, C Farquhar $^{7}$ CNZM FRCOG FRANZCOG CREI MBChB MD MPH

on behalf of the Cochrane Gynaecology and Fertility Group.

${ }^{1}$ Balliol College, University of Oxford, Oxford, United Kingdom.

${ }^{2}$ Primary Care Health Sciences, University of Oxford, Oxford, United Kingdom.

${ }^{3}$ The Institute of Applied Health Sciences, University of Aberdeen, Aberdeen, United Kingdom.

${ }^{4}$ Department of Obstetrics and Gynaecology, Máxima Medical Centre, Veldhoven, The Netherlands.

${ }^{5}$ Robinson Research Institute, University of Adelaide, South Australia.

${ }^{6}$ Centre for Biostatistics, University of Manchester, Manchester, United Kingdom.

${ }^{7}$ Cochrane Gynecology and Fertility Group, University of Auckland, Auckland, New Zealand.

Corresponding author:

\section{Prof Cindy Farquhar}

Cochrane Gynecology and Fertility Group, University of Auckland, Auckland, New Zealand.

c.farquhar@auckland.ac.nz

+6499239481

Running title:

Reducing research waste

Manuscript word count (excluding figures and tables):

1,439 words. 
45 Keywords: Outcome reporting bias, Core outcome set, Outcomes, Outcome measures, Cochrane, Systematic review

clinical research methods. There has been an acceptance that randomised controlled trials are the best way of establishing treatment effectiveness and a recognition that while single studies are useful, pooling knowledge from all available randomised trials is likely to provide the best evidence to guide clinical practice. Advances in methodology have accompanied technological innovations in gynaecology and reproductive medicine, such as assisted reproduction, assessment of male fertility, ovulation induction, and laparoscopic surgery. In particular, high quality systematic reviews have become important tools enabling evidence based health care decisions and identifying gaps in evidence. The Cochrane Gynaecology and Fertility Group has recently celebrated twenty years of preparing and publishing systematic reviews with a symposium in Oxford. With nearly a thousand authors and over two hundred reviews, we are well aware of the need for making research more efficient, accessible, and influential. This could be achieved by reducing research waste and addressing outcome reporting bias by developing and implementing core outcome sets (Williamson 2012).

Outcome reporting bias has been defined as "the selection for publication of a subset of the original recorded outcome variables on the basis of the results" (Kirkham 2010). For example, unpromising pregnancy data may be excluded from reports of subfertility trials in favour of more promising fertilisation rate comparisons. In addition to omitting outcomes from reported results it is also possible to undertake an alternative data analysis method. For a continuous outcome measure such as menstrual blood loss, authors may choose between multiple analyses 
including, but not restricted to: value at final follow-up; change from baseline; percentage change from baseline; and final value adjusted for baseline value (and for other baseline clinical factors) (Herman 2016). If measured repeatedly further possibilities arise including area under curve, time to fall below an arbitrary threshold, and many more summary statistics (Matthews 1990). Similar problems arise for categorical outcome measures.

When considering an unselected cohort of new Cochrane reviews, one third of reviews published contained at least one trial at high risk of outcome reporting bias and nearly one quarter may have overestimated treatment effects by at least twenty percent (Kirkham 2010). In trials designed to establish the superiority of a new intervention, the usual effect of outcome reporting bias will be to overstate both the magnitude and statistical significance of treatment effects. Simultaneously, less favourable comparisons may be suppressed. This has been observed for adverse event outcomes and may also be suspected where the trialists' interests lie in informally claiming equivalence (Saini 2014). Pre-specification of analyses is necessary for valid inference. Journal editors and systematic reviewers therefore need to be mindful of whether the reported outcomes and exact analyses were selected prior to data analysis (Page 2016). Regrettably, it is not uncommon for even primary outcomes to change between study planning and completion, potentially undermining the integrity of the study (Tricco 2016). Reasons for, and timing of, any changes to planned reporting should be sought from trial authors.

Our group is focusing upon the challenge of addressing the unwarranted, unhelpful, and often confusing variation in outcome collection and reporting. The variation in outcome reporting has been characterised in a number of different areas for example, assisted reproduction (Wilkinson 2016), endometriosis (Figure 1; Hirsch 2016) and heavy menstrual bleeding (Herman 2016). The development and use of a core outcome set would help to address these issues. Core outcome sets are well- 
defined, discriminatory, and feasible outcomes routinely collected and reported in randomised trials, systematic reviews, and overviews of systematic reviews (Williamson 2012). They represent a minimum data set of outcomes selected and prioritised by key stakeholders including healthcare professionals, researchers, and patients. The development and use of a core outcome set does not enforce harmony at the expense of innovation. The existence or use of a core outcome set does not imply that outcomes in a trial should be restricted (Williamson 2012). Rather, there is an expectation that the collection and reporting of core outcomes will make it easier for the results of trials to be compared, contrasted, and combined as appropriate, thus facilitating the incorporation of research findings into routine clinical practice (Williamson 2012).

Recognising that the current inconsistency in outcome reporting is a serious hindrance to progress in our specialty, eight-four editors of Women's Health journals, including the Cochrane Gynaecology and Fertility Group, have formed a consortium to support core outcome sets (Khan 2016). The Core Outcomes in Women's Health [CROWN] initiative [www.crown-initiative.org] will support the development, dissemination, and implementation of core outcome sets across our specialty. We aim to increase the value of each individual trial to ensure all trials report core outcomes and, therefore, routinely contribute data to important research questions.

Core outcome sets are currently being developed for endometriosis, fibroids, heavy menstrual bleeding, menopause, and subfertility. The Core Outcome Measures for Effectiveness Trials (COMET) initiative has performed a systematic review of methods for the derivation of core outcome sets across diverse disciplines and suggests three broad stages: [1] identifying potential core outcomes; [2] determining core outcomes using robust consensus methods engaging key stakeholders including patients; and [3] determining how core outcomes should be measure. However, to our knowledge, there is limited guidance for the most appropriate methods to develop core outcome 
sets. For example, in the absence of a standardised approach, different researchers have used different methods, perhaps including different categories of participants, limiting the number of participants, or only entering primary outcomes from trial reports into the consensus process, decisions that are rarely justified. Given the uncertainty in core outcome set development methods, further methodological research is urgently required. A research agenda could be designed through the CROWN initiative to ensure that future core outcome sets developed across our speciality are robust.

The Cochrane Fertility and Gynaecology Group brings together researchers undertaking clinical trials and observational studies in the field of reproductive medicine and benign gynaecology from around the world. We plan to utilise this opportunity to build research capacity by facilitating collaboration on a global scale, ultimately leading to robust evaluation of diagnostic and therapeutic interventions and improvements in the care women and their families receive. Our infrastructure will be leveraged to develop, implement, and disseminate research into the most important clinical questions, using robust methods and core outcome sets. We aim to foster a research environment to maximise clinical gain by ensuring that the data from all relevant studies can be used for individual patient data meta-analysis undertaken as a standard procedure as part of evidence synthesis. This can be achieved by discussion at the planning stage, collaborative applications for multinational studies, sharing, and publishing protocols. We will expand and improve the capacity and capability for clinical research within our specialty by delivering courses in research methodology and by mentoring young colleagues from across the globe.

Other opportunities exist. The performance of systematic reviews within our group provides an excellent opportunity to identify gaps in knowledge and establish research priorities. We will disseminate this information to relevant stakeholders to facilitate the development of a global research agenda, and provide a forum for 
communication between potential trialists prior to studies commencing, thereby reducing duplication and waste. Finally, we plan to proactively link with policy makers, funders, and patient organisations in individual countries, to facilitate international collaboration and interaction. We will advocate for further global programme grants utilising methods to reduce research waste including development of core outcome sets. The results of this ambitious programme of work should contribute to advancing the usefulness of research to inform clinical practice, enhance patient care, and improve patient outcomes.

Despite escalation in research activity and an exponential rise in published papers, many of the fundamental questions in subfertility and gynaecology remain. One of the key reasons for this is inherent waste due to fragmented research activity and inconsistency in the collection and reporting of outcomes (Ioannidis 2014). A global effort is urgently needed to link evidence synthesis with primary evaluative research in a concerted initiative which will deliver research which is methodologically robust, clinically meaningful, and capable of improving the quality of care. Such an initiative requires skill, confidence, leadership and above all, prioritisation of the needs of patients and society over narrow considerations of maximising research output at all costs. We are drowning in research which is singularly lacking in impact. We need fewer but better studies.

\section{Acknowledgements}

We would like to thank the thousand authors, from over 45 countries, who have contributed to two hundred reviews, and who are committed to reducing research waste; and the delegates who attended the Cochrane Gynaecology and Fertility 2016: advancing women's health through evidence meeting held at the University of Oxford, United Kingdom ( $1^{\text {st }}-3^{\text {rd }}$ April 2016). 
190 Declaration of interest

191 Dr. Duffy is a British Journal of Obstetrics and Gynaecology trainee scientific editor

192 and Cochrane Gynaecology and Fertility group editor, founding member of the Core

193 Outcomes in Women's and Newborn Health (CROWN) initiative, and has established

194 several consortiums developing and implementing core outcome sets. Prof.

195 Bhattacharya reports support from pharmaceutical companies associated with

196 fertility treatment for departmental seminars and for colleagues' attendance at

197 conferences, outside the submitted work. Prof. Vail reports non-financial support

198 from Cochrane Gynaecology and Fertility Group, grants from National Institute for

199 Health Research, outside the submitted work; and is a Statistical Editor for Cochrane

200 Gynaecology and Fertility Group (no remuneration). Mr. Wilkinson reports grants

201 from National Institute for Health Research and is a statistical editor for Cochrane

202 Gynaecology and Fertility Group. Publishing in peer-reviewed journals is beneficial to

203 his career. Prof. Farquhar reports that she is the co-ordinating editor of the Cochrane

204 Gynaecology and Fertility Group. The remaining authors report no competing

205 interests. The ICMJE disclosure forms are available as online supporting information.

207 Contribution to authorship

208 Commentary concept and design: JMD, CB, SB, MH, BM, AV, JW, and CF. Drafting of 209 the manuscript: JMD, CB, SB, MH, BM, AV, JW, and CF. Critical revision of the 210 manuscript for important intellectual content: JMD, CB, SB, MH, BM, AV, JW, and CF.

212 Funding

213 The commentary was not funded.

215 References 
Duffy JMN, van't Hooft J, Gale C, Brown M, Grobman W, Fitzpatrick R, et al. A

217 protocol for developing, disseminating, and implementing a core outcome set for 218 pre-eclampsia. Preg Hyper 2016; in press.

219 Herman M, Penninx J, Geomini P, Mol B, Bongers MY. Choice of primary outcomes

220 evaluating treatment for heavy menstrual bleeding: a systematic review. BJOG 2016;

$221 \quad 123: 1593-1598$.

222 Hirsch M, Duffy JMN, Kusznir J, Davies C, Plana M, Khan KS. Variation in outcome

223 reporting in endometriosis trials: a systematic review. AJOG 2016; 214:452-464.

224 Ioannidis J, Greenland S, Hlatky M, Khoury MJ, Macleod MR, Moher D, et al.

225 Increasing value and reducing waste in research design, conduct, and analysis. Lancet

$226 \quad 2014 ; 383: 166-175$.

227 Khan KS. The CROWN initiative: journal editors invite researchers to develop core

228 outcomes in women's health. BJOG 2014; 121:1181-1182.

229 Kirkham J, Dwan K, Altman D, Gamble C, Dodd S, Smyth R, Williamson PR. The impact

230 of outcome reporting bias in randomised controlled trials on a cohort of systematic

231 reviews. BMJ 2010; 340:c365.

232 Matthews JN, Altman DG, Campbell MJ, Royston P. Analysis of serial measurements

233 in medical research. BMJ 1990; 300:230.

234 Page MJ, Higgins JP. Rethinking the assessment of risk of bias due to selective

235 reporting: a cross-sectional study. Syst Rev 2016; 8:108.

236 Saini P, Loke Y, Gamble C, Altman D, Williamson PR, Kirkham J. Selective reporting

237 bias of harm outcomes within studies: findings from a cohort of systematic reviews.

238 BMJ 2014; 349:g6501.

239 Tricco AC, Cogo E, Page MJ, Polisena J, Booth A, Dwan K, et al. A third of systematic

240 reviews changed or did not specify the primary outcome: a PROSPERO register study.

241 J Clin Epidemiol 2016; in press.

242 Wilkinson J, Roberts SA, Showell M, Brison DR; Vail A. No common denominator: a

243 review of outcome measures in IVF RCTs. Hum Reprod 2016; in press. 
244 Williamson PR, Altman DG, Blazeby J, et al. Developing core outcome sets for clinical 245 trials: issues to consider. Trials 2012; 13:132. 
Figure 1. Outcome reporting in endometriosis trials. Largest 25 studies listed by study size reporting pain and fertility outcomes (Hirsch 2016 ).
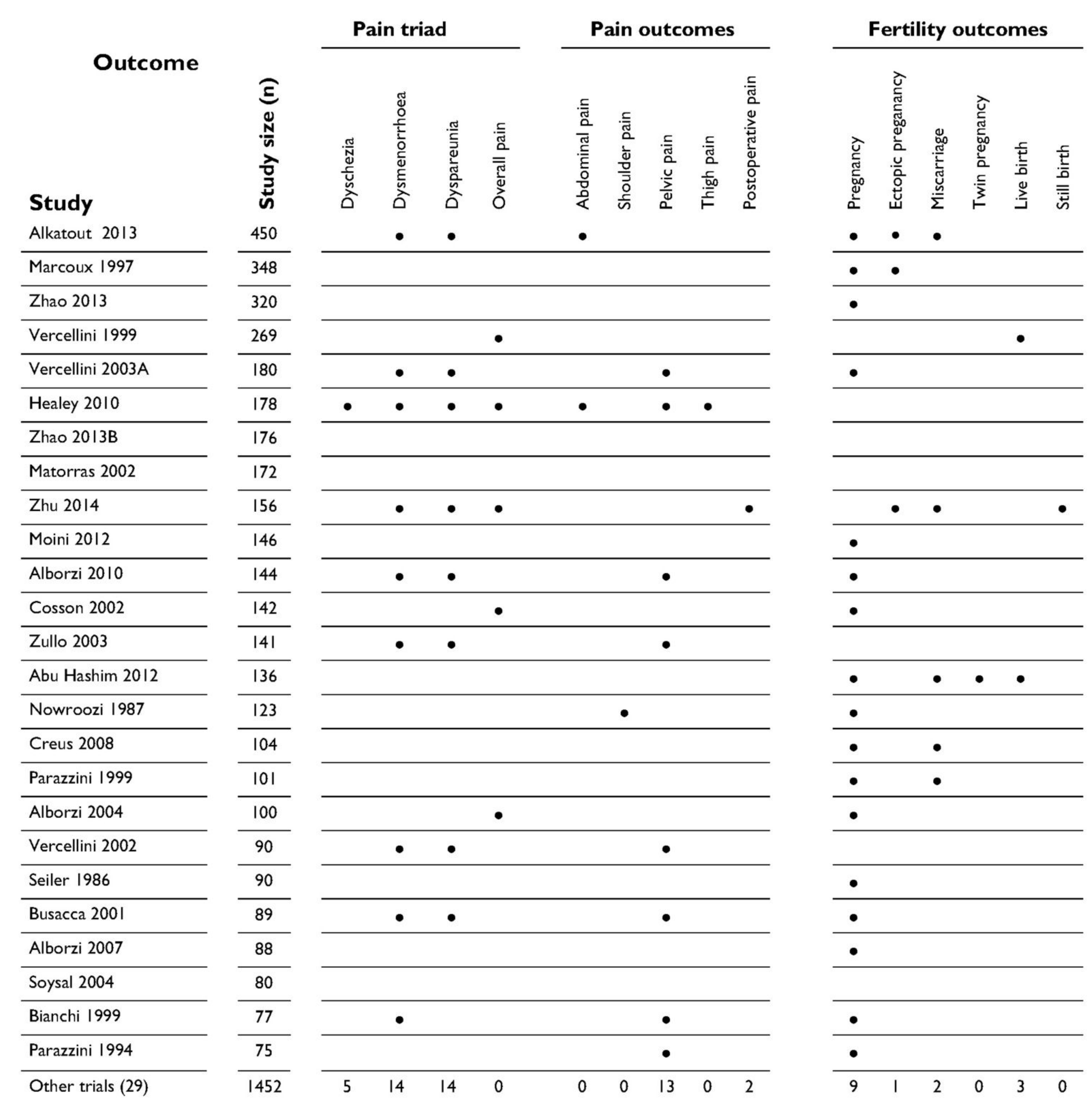

- Outcome reported in trial report 